Nota Científica

\title{
Experiencias de pesca con red de enmalle en las islas Robinson Crusoe y Santa Clara, Chile*
}

\author{
Patricio M. Arana \\ Escuela de Ciencias del Mar, Universidad Católica de Valparaíso \\ Casilla 1020, Valparaíso, Chile \\ E-mail: parana@ucv.cl
}

Recibido: 7 diciembre 1998; versión corregida: 25 mayo 1999; aceptado: 18 julio 1999

\begin{abstract}
RESUMEN Se efectuaron pescas exploratorias con red de enmalle alrededor de las islas Robinson Crusoe y Santa Clara (Archipiélago de Juan Fernández, Chile) en enero-febrero de 1997, realizándose un total de 14 caladas en diferentes lugares en torno a estas islas, obteniendo una captura total de $599 \mathrm{~kg}$ en 106 horas de reposo. Los principales recursos capturados según su aporte en peso correspondieron al tollo de Juan Fernández, Squalus mitsukurii (53,9\%); jurel de Juan Fernández, Pseudocaranx chilensis (19,9\%); corvina de Juan Fernández, Umbrina reedi (7,8\%); vidriola, Seriola peruanus (7,3\%); jerguilla de Juan Fernández, Girella albostriata (7,2\%); y, otras especies (3,8\%). Mediante estas experiencias se demuestra que este arte puede ser calado con relativa facilidad y seguridad en diversos lugares alrededor de estas islas. Además, los niveles de captura obtenidos hacen atractiva su utilización con el fin de obtener peces, la mayoría de los cuales son especies de alta calidad, que tuvieron tamaños y pesos requeridos para consumo humano o para ser empleados como carnada en las trampas langosteras.
\end{abstract}

Palabras claves: pesca, peces, red de enmalle, archipiélago de Juan Fernández, Chile.

\section{Fishing with gillnets in Robinson Crusoe and Santa Clara islands, Chile}

ABSIRACT. The gillnet fishing survey was conducted around Robison Crusoe and Santa Clara islands (Juan Fernandez archipelago, Chile) in January-February 1997. A total of 14 hauls at different locations around these islands were taken, with a total catch of $599 \mathrm{~kg}$ in 106 hours of soak. The main resources caught sorted by weight were: Juan Fernandez catshark, Squalus mitsukurii (53.9\%); Juan Fernandez mackerel, Pseudocaranx chilensis (19.9\%); Juan Fernandez croaker, Umbrina reedi (7.8\%); yellowtail, Seriola peruanus (7.3\%); Juan Fernandez opaleye, Girella albostriata (7.2\%); and, other species (3.8\%). These experiences showed that this gear can be easily and securely set in different places around these islands. Besides, the catch levels obtained makes its use quite attractive for the purpose of obtaining fishes, most of which are species of high quality, and have the appropriate size and weight required for human consumption or for to be used as bait in lobster traps.

Key words: fishing, fishes, gillnet, Juan Fernandez archipelago, Chile.

\footnotetext{
* Artículo generado como parte del "Programa de pesca exploratoria y experimental de recursos pesqueros alternativos a la langosta en las islas Robinson Crusoe y Santa Clara" (Proyecto FIP 95/25), financiado por el Fondo de Investigación Pesquera y realizado por la Universidad Católica de Valparaíso.
} 
El archipiélago de Juan Fernández (3340'S$\left.78^{\circ} 50^{\prime} \mathrm{W}\right)$, a 365 millas náuticas de la costa central de Chile, está conformado por las islas Robinson Crusoe, Santa Clara y Alejandro Selkirk, siendo la primera de ellas la única con población permanente, constituida principalmente por pescadores artesanales que operan en ese archipiélago. La economía de estas islas está sustentada primordialmente por la pesca de la langosta de Juan Fernández (Jasus frontalis H. Milne-Edwards, 1837) y en segundo lugar por un turismo incipiente. La explotación de este crustáceo es una de las actividades pesqueras más antiguas del país y cuenta con medidas de manejo desde comienzos del siglo XX. No obstante, en los últimos años se ha observado una importante reducción en los desembarques de esta especie, alcanzando alrededor de 23 toneladas anuales en el quinquenio 1990-1994, cifra notablemente inferior al récord de 134 ton registrado en 1952.

Por razones de ordenamiento pesquero, las faenas extractivas de la langosta están permitidas en las islas Robinson Crusoe y Santa Clara entre el 1 de octubre y 14 de mayo del año siguiente, obligando a los pescadores a paralizar sus actividades laborales durante la vigencia de la veda. En la actualidad se cuenta con antecedentes acerca de la presencia de ciertos recursos de interés pesquero que no son explotados en estas islas y que podrían contribuir a generar beneficios a la población insular (Arana, 1987; Arana y Ziller, 1985). Algunas especies son costeras o demersales y otras pelágicas migratorias, que visitan el archipiélago en ciertos períodos del año.

Por los antecedentes expuestos, se considera importante identificar y desarrollar alternativas de explotación pesquera, que permitan a los pescadores efectuar actividades diferentes o complementarias a las realizadas tradicionalmente. El presente trabajo estuvo orientado a investigar la factibilidad de utilizar redes de enmalle alrededor de estas islas, para establecer qué recursos ícticos podrían ser capturados con el empleo de este arte de pesca.

Para realizar las faenas de pesca con este arte, se utilizaron las chalupas de alta mar $\mathrm{N}^{\circ} 24$ "Libertad" y $\mathrm{N}^{\circ} 65$ "Matilde". La red utilizada correspondió a un típico arte de enmalle utilizado por el subsector artesanal, armado con paños de poliamida monofilamento de $0,6 \mathrm{~mm}$ de diámetro y un claro de malla de $15 \mathrm{~cm}$, utilizando 3 paños de 38,5 brazas de largo cada uno, con una longitud total de 155,5 brazas $(210 \mathrm{~m})$ y una altura teórica de 6 brazas $(10,9$ m) (Fig. 1), aparejo que no es utilizado por los pescadores de este archipiélago. El calado se realizó por lo general en forma paralela a la costa a diferentes profundidades.

La captura fue separada por especie y a cada ejemplar se le determinó in situ el sexo y le fue medida su longitud total en un ictiómetro, registrándose esta medida al centímetro inferior. Además, se pesaron individualmente los organismos mediante un dinamómetro con capacidad máxima de $12 \mathrm{~kg}$ y precisión de $+250 \mathrm{~g}$. Seguidamente, a un cierto número de los ejemplares capturados se les midió el perímetro máximo antes de la presencia de aletas o apéndices, utilizando para ello una cinta métrica, anotando la medida redondeada al centímetro. El disponer de esta medidas permitió la utilización de la metodología propuesta por MacCombie y Berst (1969), que consiste en establecer la relación entre el perímetro máximo de la presa y el perímetro interno de la malla empleada.

Finalmente, con el objeto de establecer la identidad de la fauna íctica capturada con los espineles, se procedió a recolectar y preservar en formaldehído un cierto número de ejemplares de cada especie. La labor de identificación fue realizada posteriormente por un especialista.

Entre enero y febrero de 1997 se efectuaron 14 caladas con este arte, en diferentes lugares en torno a las islas Robinson Crusoe y Santa Clara. Usualmente, se caló la red de enmalle al atardecer y se viró al amanecer del día siguiente. En dichas experiencias se obtuvo una captura total de $599,2 \mathrm{~kg}$ en 105,75 horas de reposo (Tabla 1). Los rendimientos por lance de pesca estuvieron comprendidos entre 0 y $262 \mathrm{~kg}$, con una media global de $46 \mathrm{~kg} /$ lance o 5,7 $\mathrm{kg} / \mathrm{hora}$ de pesca.

Los recursos más relevantes, en peso fueron el tollo de Juan Fernández (Squalus mitsukurii Jordan y Snyder, 1903) (53,9\%), jurel de Juan Fernández (Pseudocaranx chilensis Guichenot, 1848) (19,9\%), corvina de Juan Fernández (Umbrina reedi Günther, 1880) (7,8\%), vidriola (Seriola peruanus Steindachner, 1881) $(7,3 \%)$ y jerguilla de Juan Fernández (Girella albostriata Steindachner, 1898) (7,2\%). Además de los mencionados recursos, se capturaron otros peces en menor cantidad, totalizando sólo 22,9 kg (3,8\%). Entre éstos se encontraron lenguados (Paralichthys fernandezianus Steindachner, 1905), jurel continental (Trachurus murphy Nichols, 1920) y bacalao de Juan Fernández (Polyprion oxygeneios Schneider, 1801). Todas es- 
tas especies son conocidas y componentes comunes de la fauna íctica de estas islas (Sepúlveda y Pequeño, 1985; Sepúlveda, 1987).

Los ejemplares retenidos fueron de tamaños y pesos atractivos para su utilización como pesca blanca o ser empleados como carnada en las trampas langosteras (Tabla 2). Es posible que con un mayor conocimiento de los caladeros de pesca se obtengan capturas superiores a la media observada, en espe- cial de vidriola en los períodos cuando arriba en abundancia a estas islas (primavera-verano).

El perímetro máximo de los ejemplares capturados estuvo comprendido entre 23 y $40 \mathrm{~cm}$ (Tabla 3 ). El tollo de Juan Fernández fue el recurso más importante con un perímetro máximo promedio de $30,1 \pm 0,76 \mathrm{~cm}$, relacionado adecuadamente con los $30 \mathrm{~cm}$ de perímetro teórico de las mallas utilizadas en la red. De acuerdo a la metodología de

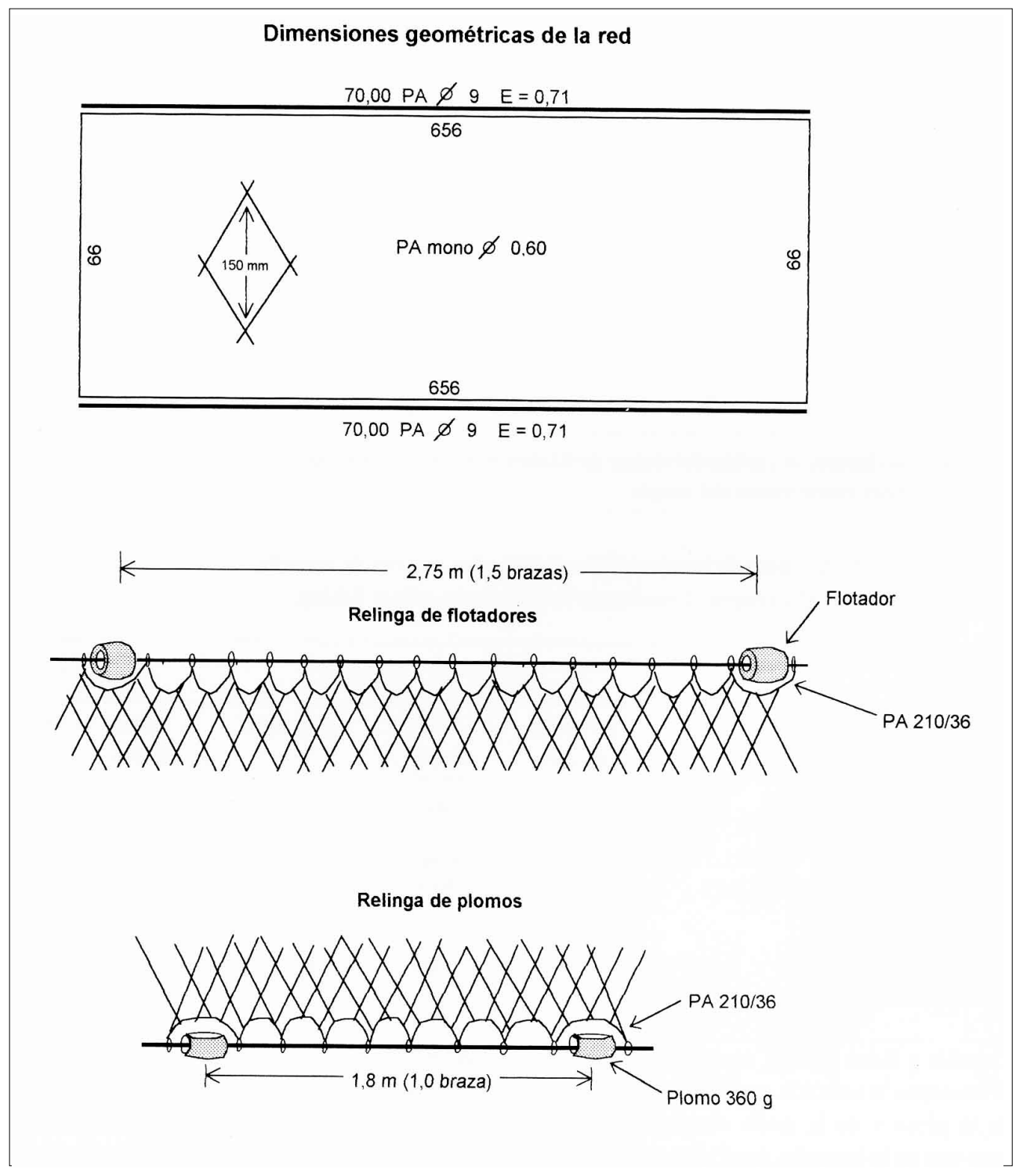

Figura 1. Esquema general de los componentes y dimensiones principales de la red de enmalle utilizada en las islas Robinson Crusoe y Santa Clara.

Figure 1. General scheme of components and main dimensions of the gillnet used around Robinson Crusoe and Santa Clara islands. 
Tabla 1. Capturas obtenidas con red de enmalle, alrededor de las islas Robinson Crusoe y Santa Clara. Table 1. Catches obtained in gillnet fishing survey around Robinson Crusoe and Santa Clara islands.

\begin{tabular}{|c|c|c|c|c|c|c|c|c|c|}
\hline \multirow{2}{*}{ Lance $\mathbf{N}^{\mathbf{0}}$} & \multirow{2}{*}{$\begin{array}{l}\text { Tiempo de } \\
\text { reposo (hr) }\end{array}$} & \multicolumn{7}{|c|}{ Captura por recurso $(\mathbf{k g})$} & \multirow[t]{2}{*}{ Total } \\
\hline & & $\begin{array}{c}\text { Corvina } \\
\text { de J.F. }\end{array}$ & $\begin{array}{c}\text { Jerguilla } \\
\text { de J.F. }\end{array}$ & $\begin{array}{c}\text { Jurel de } \\
\text { J.F. }\end{array}$ & Lenguado & $\begin{array}{c}\text { Tollo } \\
\text { de J.F. }\end{array}$ & Vidriola & Otros & \\
\hline 1 & 2,5 & 7,5 & - & - & - & 13,0 & 12,9 & - & 33,4 \\
\hline 2 & 4,3 & - & 7,0 & 20,9 & - & - & 22,5 & - & 50,4 \\
\hline 3 & 1,0 & - & - & - & - & - & - & - & 0,0 \\
\hline 4 & 2,0 & - & - & 2,8 & - & - & - & - & 2,8 \\
\hline 5 & 4,0 & 33,0 & 7,8 & - & - & - & - & - & 40,8 \\
\hline 6 & 7,0 & - & - & 10,4 & 0,8 & 20,0 & - & - & 31,2 \\
\hline 7 & 11,0 & - & - & 12,3 & 4,3 & 27,5 & 8,3 & 6,0 & 58,4 \\
\hline 8 & 12,0 & - & - & 6,6 & 1,8 & - & - & - & 8,4 \\
\hline 9 & 11,0 & - & - & 21,7 & 1,0 & 11,5 & - & - & 34,2 \\
\hline 10 & 12,5 & - & - & 14,3 & 1,0 & - & - & - & 15,3 \\
\hline 11 & 6,5 & - & - & 7,2 & - & 33,0 & - & 3,0 & 43,2 \\
\hline $12 *$ & 1,5 & - & - & - & - & - & - & - & $0,0^{*}$ \\
\hline 13 & 14,5 & - & 5,0 & 7,0 & - & 7,0 & - & - & 19,0 \\
\hline 14 & 16,0 & 6,1 & 23,6 & 16,2 & 5,0 & 211,2 & - & - & 262,1 \\
\hline Total & 105,8 & 46,6 & 43,4 & 119,4 & 13,9 & 323,2 & 43,7 & 9,0 & 599,2 \\
\hline \multicolumn{2}{|c|}{ Porcentaje (\%) } & 7,8 & 7,2 & 19,9 & 2,3 & 53,9 & 7,3 & 1,5 & 100,0 \\
\hline
\end{tabular}

Nota: La tabla no incluye la captura incidental de 3 lobos finos de Juan Fernández (lance No 8)

(*) Se vira la red por fuerte viento del sureste

Tabla 2. Rangos de talla y peso de los ejemplares capturados con red de enmalle.

Table 2. Length and weight ranges of specimens caught in the gillnet fishing.

\begin{tabular}{|l|cccccc|}
\hline \multirow{2}{*}{ Observación } & \multicolumn{7}{|c|}{ Recurso } \\
\cline { 2 - 7 } & $\begin{array}{c}\text { Corvina } \\
\text { de J.F. }\end{array}$ & $\begin{array}{c}\text { Jerguilla } \\
\text { de J.F. }\end{array}$ & $\begin{array}{c}\text { Jurel } \\
\text { de J.F. }\end{array}$ & Lenguado & Tollo & Vidriola \\
de J.F. & \\
\hline Número de ejemplares & 13 & 23 & 48 & 11 & 70 & 17 \\
Rango de talla (cm) & $42-77$ & $40-55$ & $47-67$ & $39-58$ & $67-110$ & $50-84$ \\
Talla media (cm) & 61,3 & 47,4 & 59,3 & 46,2 & 92,6 & 63,2 \\
Rango de peso (kg) & $1-7,5$ & $1,5-2,5$ & $1,3-3,2$ & $0,8-1,8$ & $2,9-8,0$ & $1,5-4,5$ \\
Peso medio (kg) & 3,59 & 1,89 & 2,49 & 1,26 & 4,62 & 2,58 \\
\hline
\end{tabular}

MacCombie y Berst (1969), en el caso del tollo de Juan Fernández la relación entre el perímetro máximo de la presa y de la malla correspondió a 1,0, mientras que en la jerguilla, jurel y lenguado de Juan Fernández, la relación estuvo comprendida entre 0,94 y 1,14 (Tabla 3 ).

En general, la probabilidad que un pez sea capturado en una red de pared depende de la posibili- dad que se encuentre con el arte y su habilidad para detectarlo y evadirlo. Esto puede estar relacionado con las características de la red utilizada, visión de los organismos, transparencia del agua y principalmente, de la capacidad de natación de los peces, que a su vez está relacionado con su longitud (Regier y Robson, 1966).

De acuerdo a McCombie y Fry (1960), el perí- 
metro máximo proporciona el mejor estimador de la talla de los ejemplares que se pueden capturar con un tamaño particular de malla, mientras que Marais (1985) considera que la forma y los apéndices de las diferentes especies influye en la retención de los peces. Sobre este particular, Hamley (1975), Hovgard (1996), y Reis y Pawson (1999) determinaron que no sólo influye en la retención el perímetro máximo de los peces y los apéndices, sino también el lugar específico del cuerpo en que queda retenido en la malla. De allí que Regier (1969) considera que es más preciso medir el perímetro de los peces en la parte donde son enmallados que su longitud, dada la variabilidad entre la talla y el perímetro de los organismos. En este trabajo, el considerar el perímetro máximo antes de aletas o apéndices entregó un estrecha relación con el tamaño de la malla utilizada.
En el lance 8 realizado el 24 de enero de 1997, se capturaron tres lobos finos de Juan Fernández (Arctocephalus philipii Peters, 1866), lo que provocó que la red se enredara y no operara en forma adecuada. Uno de los lobos se encontró muerto, mientras que los otros dos fueron liberados sin daño aparente. Por esta razón, se debe tener especial cuidado de desplegar este arte alejado de aquellos lugares donde se conoce la existencia de loberías o se observa la presencia de lobos finos de Juan Fernández, ya que puede provocar mortalidad incidental en estos mamíferos. Al respecto, los lobos finos de Juan Fernández son abundantes cerca de las bahías Vaquería, Tres Puntas, Carvajal y El Padre.

Los resultados indican que este arte es de operación simple, pudiendo ser calado con facilidad en diversos lugares alrededor de las islas, principalmente en bahías y sectores protegidos. No obstante, se

Tabla 3. Perímetro máximo de los ejemplares capturados con red de enmalle.

Table 3. Maximum girth observed in specimens caught in the gillnet fishing.

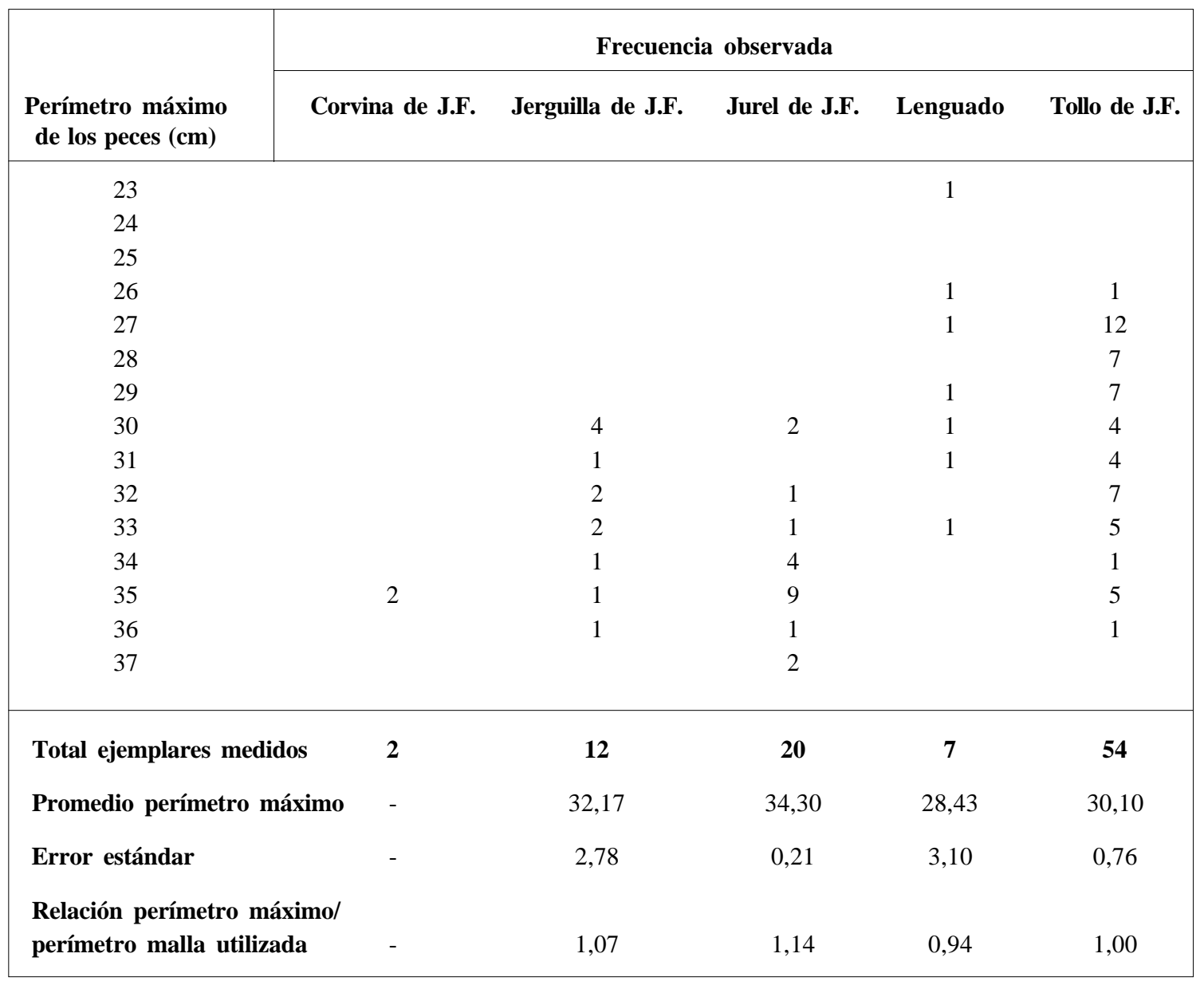


deben evitar los fondos irregulares que pudieran provocar enredos; así, también, se recomienda calar la red en bahías protegidas del viento y de las corrientes, sobre fondos de arena o piedras. Con algo de experiencia es posible seleccionar caladeros para este arte, ya sea respecto a la época del año que es más favorable su uso, como los lugares donde se obtienen mejores rendimientos y determinadas especies.

Si bien se efectuó una cantidad reducida de lances, es posible esperar cierto nivel de captura que hace atractiva la utilización de redes de enmalle en la obtención de recursos ícticos, algunos de excelente calidad, como la vidriola, corvina, lenguados y jerguilla de Juan Fernández (Fig. 2). Además, todos los ejemplares capturados fueron de gran talla, lo que indica que el tamaño de malla empleado es apropiado para su uso en este lugar. Así, los peces de mayor calidad se podrían destinar a consumo humano local o exportados al continente, y los de menor calidad como carnada de las trampas langosteras, lo que facilitaría las labores diarias de los pescadores, que requieren utilizar parte importante de su tiempo en asegurar el cebo para sus trampas.

No obstante, las ventajas de la posible de redes de enmalle en estas islas debería ser cuidadosamente regulado en su número, dimensiones y tamaño de las mallas empleado en la red. Así, también, se debería observar cuidadosamente el impacto que tendría su utilización sobre la fauna marina.

\section{AGRADECIMIENTOS}

El autor agradece la colaboración brindada por las tripulaciones de las chalupas de alta mar "Libertad" y "Matilde" con las cuales se efectuaron las experiencias y al Sr. Luis González por aportar su experiencia en el armado y operación de redes de enmalle. Así, también, se agradece muy especialmente al Dr. Eduardo de la Hoz Urrejola (Q.E.P.D.), de la Universidad Católica de Valparaíso, por su aporte en la identificación de las especies capturadas con este arte de pesca.

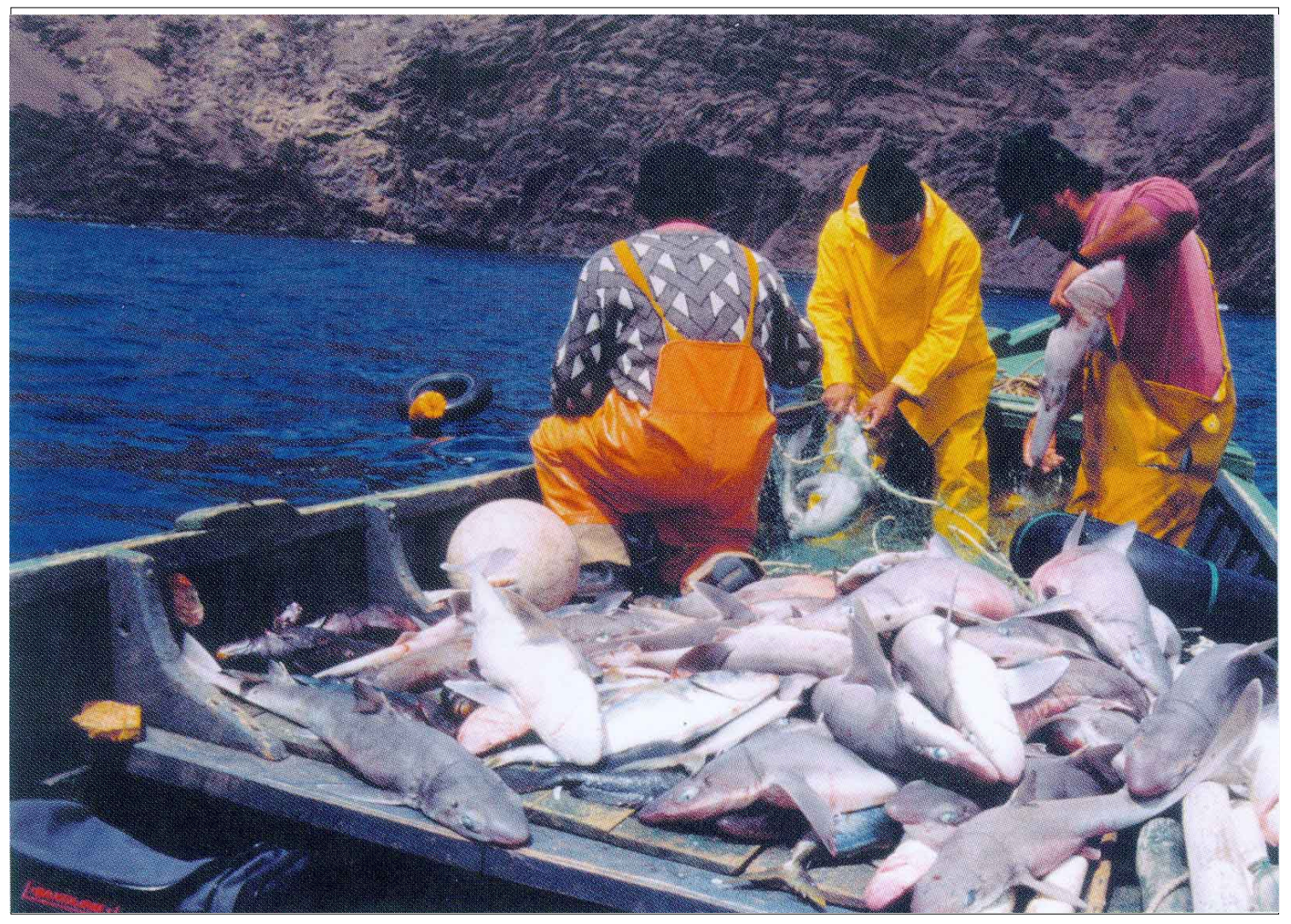

Figura 2. Captura de peces y tiburones obtenida con red de enmalle.

Figure 2. Fish and shark catches obtained with gillnets. 


\section{REFERENCIAS}

Arana, P. y S. Ziller. 1985. Antecedentes generales sobre la actividad pesquera realizada en el archipiélago de Juan Fernández. En: P. Arana (ed.). Investigaciones Marinas en el Archipiélago de Juan Fernández. Esc. Ciencias del Mar, UCV, Valparaíso, 125-152.

Arana, P. 1987. Perspectivas históricas y proyecciones de la actividad pesquera realizada en el archipiélago de Juan Fernández, Chile. En: J.C. Castilla (ed.). Islas Oceánicas Chilenas: Conocimiento Científico y Necesidades de Investigaciones. Edic. Univ. Católica de Chile, 319-353.

Hamley. J.M. 1975. Review of gillnet selectivity. J. Fish. Res. Bd. Can., 32(11): 1943-1969.

Marais, J.F.K. 1985. Some factors influencing the size of fishes caught in gillnets in eastern Cape estuaries. Fish Res., 3: 251-261.

McCombie A.M. y A.H. Berst. 1969. Some effects of shape and structure of fish on selectivity of gillnets. J. Fish. Res. Bd. Can., 26: 2681-2689.
Sepúlveda, J.I. 1987. Peces de las islas oceánicas chilenas. En: J.C. Castilla (ed.), Islas Oceánicas Chilenas: Conocimiento Científico y Necesidades de Investigaciones. Edic. Univ. Católica de Chile, 225245.

Sepúlveda, J.I. y G. Pequeño. 1985. Fauna íctica del archipiélago de Juan Fernández. En: P. Arana (ed.). Investigaciones Marinas en el Archipiélago de Juan Fernández. Esc. Ciencias del Mar, UCV, Valparaíso, 81-91.

Regier, H.A. 1969. Fish size parameters useful in estimating gill net selectivity. Prog. Fish Cult., 31(1): 57-59.

Regier, H.A. y D.S. Robson. 1966. Selectivity nets, especially to lake whitefish. J. Fish. Res. Bd. Can., 23(3): 423-454.

Reis, E.G. y M.G. Pawson. 1999. Fish morphology and estimating selectivity by gillnets. Fish. Res., 39: 263-273. 